\title{
OPTIMIZING LOCALIZATION ROUTE USING PARTICLE SWARM-A GENETIC APPROACH
}

\author{
${ }^{1}$ Lakshmanan, L. and ${ }^{2}$ D.C. Tomar \\ ${ }^{1}$ Faculty of Computer Science and Engineering, Sathyabama University, Chennai, India \\ ${ }^{2}$ Department of IT, Jerusalem College of Engineering, Chennai, India
}

Received 2013-09-24; Revised 2014-01-02; Accepted 2014-01-20

\begin{abstract}
One of the most key problems in wireless sensor networks is finding optimal algorithms for sending packets from source node to destination node. Several algorithms exist in literature, since some are in vital role other may not. Since WSN focus on low power consumption during packet transmission and receiving, finally we adopt by merging swarm particle based algorithm with genetic approach. Initially we order the nodes based on their energy criterion and then focusing towards node path; this can be done using Proactive route algorithm for finding optimal path between Source-Destination (S-D) nodes. Fast processing and pre traversal can be done using selective flooding approach and results are in genetic. We have improved our results with high accuracy and optimality in rendering routes.
\end{abstract}

Keywords: Particle Swarm Optimization, Wireless Sensor Network, Proactive Routing, Reactive Routing, Genetic

\section{INTRODUCTION}

One of the most vital problems in wireless sensor networks is finding optimal routes for transmitting data between sources to destination. Generally WSN node pairs in a multi-hop fashion. Several algorithms have been proposed for routing in WSN. A new family of algorithms inspired by Swarm Intelligence (SI) which provides a novel approach to distributed optimization problems. The expression "swarm intelligence" defines any attempt to design algorithms enthused by the collective behaviour of social insect colonies (Ant(ACO) and Bee Colony (BCO)) and another animal societies(Fish colony optimization). Swarm intelligence provides a basis with which, it is possible to explore distributed optimization problems without exploring centralised control or provision of a global model in WSN routing strategy. Initial research has unveiled a great deal of matching properties between the routing requirements of sensor networks and certain feature of SI. There are some notable routing algorithms in WSN which uses ant like mobile agents to maintain routing and topology to discover optimal path for Wireless Sensor Corresponding Author: Lakshmanan, L., Faculty of Computer Science and Engineering, Sathyabama University, Chennai, India
Network (WSN). In this study we order the nodes based on their energy competence and its focus towards node path (Osama et al., 2005).

Swarm intelligence boasts a number of advantages due to the usage of mobile agents they were denoted as follows:

- Scalability: Population of the agents can be adapted consistently based on the network size. Scalability is also promoted by local and scattered agent interactions

- Fault tolerance: Swarm intelligence processes do not rely on a centralized control mechanism. Therefore the loss of a few nodes or links does not result in shattering failure, but rather leads to graceful, scalable degradation (Murthy and Manoj, 2004)

- Adaptation: Agents can alter, expire or replicate, according to network changes

- Speed: Changes in the network can be propagated very fast, in contrast with the Bellman-Ford algorithm (Clerc and Kennedy, 2002)

- Modularity: Agents act independently of other network layers (Tanese, 1989)

- Autonomy: Little or no human supervision is required (Lee and Knignt, 2005) 
- Parallelism: Agent operations are inherently parallel. These properties make swarm intelligence very attractive for ad-hoc wireless networks. They also render swarm intelligence suitable for a variety of other applications, apart from routing, including robotics (Robinson et al., 2002; Mussetta et al., 2004; 2005; Juang, 2004) and optimization (Rong et al., 2005; Visu et al., 2012).

\subsection{Particle Swarm Optimization (PSO)}

\subsubsection{A. General PSO Algorithm}

PSO is a population based optimization technique, developed by (Kennedy, 1997; Rahmat-Samii and Michielssen, 1999; Arabas et al., 1994), inspired by social behavior of Ants, bird flocking (and schools of fish). In PSO, a swarm is a collection of particles where each particle has both a position and velocity. The position of the particle represents a candidate solution to the problem space while the velocity is used to move the particle from one position to another. The "classical" PSO equation where the position and velocity represents physical attributes of the particles is represented by ( 1 and 2$)$ :

$$
\begin{aligned}
& \mathrm{V}_{\text {id }}=\mathrm{V}_{\text {id }}+\mathrm{n}_{1} \mathrm{r}_{1}\left(\mathrm{P}_{\text {id }}-\mathrm{X}_{\text {id }}\right)+\mathrm{n}_{2} \mathrm{r}_{2} \\
& \left(\mathrm{P}_{\text {id }}-\mathrm{X}_{\text {id }}\right) \mathrm{i}=1,2 \ldots, \mathrm{N} ; \mathrm{d}=1,2, . . \mathrm{D} \\
& \mathrm{X}_{\text {id }}=\mathrm{X}_{\text {id }}+\mathrm{V}_{\text {id }}
\end{aligned}
$$

$P_{\text {id }}$ is the personal best position, a particle has reached; $P_{i d} n$ is the global best position of all the $n$ particles. $\eta 1$ (the self-confidence factor) and $\eta 2$ (the swarm-confidence factor) are positive constants called 'acceleration constants' to determine the influence of $P_{\text {id }}$ and $P_{i d} n ; r_{1}$ and $r_{2}$ are independent random numbers in the range $[0,1] . \mathrm{n}$ is the total number of particles in the swarm and D is the dimension of the problem search space. PSO starts by randomly initializing the position and velocities of all the particles in the swarm over the problem space. The position of $i^{\text {th }}$ particle is represented by the vector $X_{i}=\left[X_{i 1}, X_{i 2} \ldots X_{i d}\right]$ and velocity of $i^{\text {th }}$ particle is represented by the vector $V_{i}=\left[V_{i 1}, V_{i 2} \ldots V_{i d}\right]$, where $\mathrm{d}$ is the number of function parameters being optimized. For each iteration (until the convergence criteria is met), the fitness function is applied to the particles to quantize their respective positions over the problem search space (Ciuprina et al., 2002). The particle among the finest fitness value in the neighborhood is marked as the global/local best particle.
Each particle will also keep a record of its personal best position searched so far. Equation (1) is used to calculate new velocity for each particle in the swarm based on particle's preceding velocity, its current and personal finest position and the position of the particle with best fitness value in the neighborhood. Equation (2) is then used to apply the velocity to the particle. As an outcome of this, the particle will move regarding to a new position i.e., it will now correspond to a new aspirant resolution to the problem being studied.

\subsection{Genetical Swarm Optimization}

Some comparison performances of GA and PSO are present in literature (Grimaccia et al., 2006) underlining the reliability and convergence speed of both methods, but continuing strategy tends in keeping them separate. Anyway, the population-based representation of the parameters that characterize a particular solution is the same for both the algorithms; therefore it is possible to implement a hybrid technique in order to utilize the qualities and uniqueness of the two algorithms. Some attempts have been done in this direction (Visu and Kannan, 2013), with good results.

The hybrid technique here proposed, called Genetic Swarm Optimization (GSO), is essentially a population-based heuristic search technique which can be used to solve combinatorial optimization problems, modelled on the concept of natural selection but also based on cultural and social evolution.

GSO is generally heuristic in nature but with weak integration of the two strategies, leads algorithm mainly used as the pre-optimizer for the initial population of the other one. GSO algorithm performs consistently in a strong cooperation with GA over PSO, since it maintains the integration of the two techniques for the entire run. In each iteration, in fact, the population is divided into two parts and they are evolved with the two techniques in pre traversal order.

They are then remerged in the modernized population, that is yet again divided randomly into two parts in the next iteration for another run of genetic or particle swarm operators.

The population revised concept can be effortlessly understood by thinking that a part of those individuals is substituted by newly generated ones by the resources of GA, while enduring the same of the earlier generation but moved on the solution space by PSO.

Since the result obtained by these techniques were heuristic at real time engagement and optimal at their best case. The correlation value has been estimated for GA, PSO, GA-PSO algorithms where GA-PSO is optimal at best run. 


\section{MATERIALS AND METHODS}

\subsection{Related Work}

Evolutionary optimization schemes like Genetic Algorithms (GA) and PSO have successfully been used in the past decade to solve many NP-hard optimization problems (Tanese, 1989) and global routing issues. GA and PSO are similar in the way that both techniques are population based search schemes that mimic the natural biological evolution and/or the social behavior of species Tanese (1989) and Kennedy (1997) which was discussed in the section I (Introduction). Each member in the population represents a candidate solution to the problem addressed and over time they evolve to represent some other candidate solution.

One advantage of PSO over GA is that PSO is more computationally efficient (Boeringer and Werner, 2004). Some performance comparison between GA and PSO have been reported in (Tanese, 1989; Boeringer and Werner, 2004; Robinson et al., 2002; Mussetta et al., 2004). Goldberg (1989), a novel GA based scheme is proposed to solve dynamic RWA problem in wavelength routed optical networks. Genetic algorithms with swarm intelligence inspired search schemes based on the idea of natural selection and natural genetics. Goldberg (1989), a member of the population (gene) represents a route from source to destination node i.e., a candidate solution to the routing sub-problem for DRWA.

Genetic operators like crossover, mutation and then selection are applied to create a new population of genes. Mussetta et al. (2005) have proposed a novel hybrid algorithm based on PSO and noising meta-heuristic for computing shortest paths in the WS network. The hybrid PSO based scheme shows better performance as compared to GA-based search algorithms for optimal shortest path computation (Mussetta et al., 2005; AlfassioGrimaldi et al., 2004; Goldberg, 1989), GA algorithms are proposed for solving DRWA in all-optical WDM networks.

In our study, the GA based schemes proposed in AlfassioGrimaldi et al. (2004) and Goldberg (1989) are merged and selected as the schemes for performance comparison purposes with our novel PSO-based algorithm.

\subsection{PSO-GA}

The hybrid technique here proposed called PSO-GA, which integrates PSO and GA algorithm for better performance. The policy behinds the PSO and GA are same as off. Since a pre traversal technique is used in order to traverse the source localization field to reduce the estimation of errors. Initially all the nodes in the Wireless Sensor Networks (WSN) are flooded with local temporal value with its HC factor. The driving constraint of GSO algorithm is the Hybridization Coefficient (HC); it express the percentage of population, each iteration is evolved with GA: So $\mathrm{HC}=0$ means the procedure is a pure PSO (the whole population is updated according to PSO operators), $\mathrm{HC}=1$ means pure $\mathrm{GA}$, while $0<\mathrm{HC}<1$ means that the corresponding percentage of the population is developed by GA, the rest with PSO. When the HC factor value is around $0.486-0.789$ then the value will be flooded based on selective flooding approach.

\subsection{Flooding in GA}

The driving constraint of GSO algorithm is the Hybridization Coefficient (HC); it express the percentage of population that in each iteration is evolved with GA: So $\mathrm{HC}=0$ means the procedure is a pure PSO (the whole population is updated according to PSO operators), $\mathrm{HC}=1$ means pure GA, while $0<\mathrm{HC}<1$ means that the corresponding percentage of the population is developed by GA, the rest with PSO.

\section{The Pseudo Code for the PSO-GA Algorithm:}

Function GA $=$ PSO (F, fit, i, m, h)

Begin

Initialize particle

do

For each particle

Calculate fitness function of the particle $i(m)$

If $i(m))$ is better than Ffit

set current value as the new Ffit

End For

Set hfit to the best fitness of $\forall$ particles

For $\forall$ particle

Calculate particle rate according $\mathrm{V}_{\mathrm{id}}=\mathrm{V}_{\mathrm{id}}+\mathrm{n}_{1} \mathrm{r}_{1}\left(\mathrm{P}_{\mathrm{id}}{ }^{-}\right.$ $\left.\mathrm{X}_{\mathrm{id}}\right)+\mathrm{n}_{2} \mathrm{r}_{2}\left(\mathrm{P}_{\mathrm{id}}-\mathrm{X}_{\mathrm{id}}\right)$

Update particle position according equation $X_{i d}=X_{i d}$ $+\mathrm{V}_{\text {id }}$

End_For

Check $\forall$ particle

For $\forall$ iterationGenerate Local criterian for hfit

Set Ffit for maximum

Calculate connectionMatrix

Calculate Fitratio,estimation error

End_While when maximum recursions attained End

\section{RESULTS AND DISCUSSION}

\subsection{Simulation Results}

The results are shown in Fig. 1. In this figure, the estimated position of PSO algorithm is much closed to 
the actual source position, the estimation error between the actual position and the estimated position is about $1.3 \mathrm{~m}$. The estimated position will approach to the actual position with the increasing number of sensor nodes and signal-noise-ratio through the information of experiments and while the number of sensor nodes is small the estimated position of PSO algorithm is more accurately than other searching algorithms.

Extensive simulation has been conducted to compare the performance of the proposed PSO-GA algorithm to other energy-based source localization algorithms (GA and PSO) using Mat lab. We conducted 1000 repeated trials with equal intervals of 20 ts (timeslot) and the average value is to estimate localization error in initial route path. All three energybased source localization methods such as (Particle swarm optimization-Genetic Approach algorithm, MultiResolution searching (MR) and Exhaustive Searching (ES) algorithm) are used tocalculate the source localization and the error is recorded in each trial.

We have performed lots of simulations to examine the performance of the proposed algorithm and other two algorithms. Different sensors and signal-noise-ratio will affect the localization error. The relationship between the estimation error of three localization algorithms and node number is shown in Fig. 2 and the relationship between the estimation error of three localization algorithms and signal-noise-ratio is shown in Fig. 3.

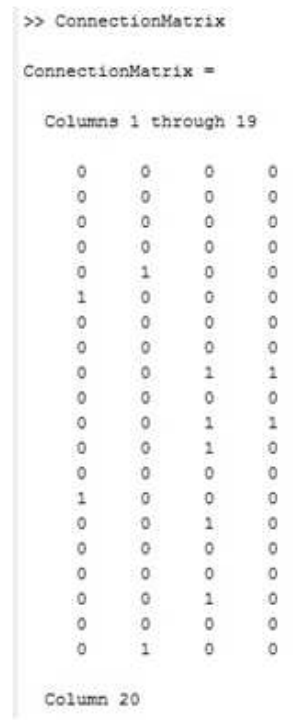

Fig. 1. Connection matrix for estimation of error for PSO-GA algorithms

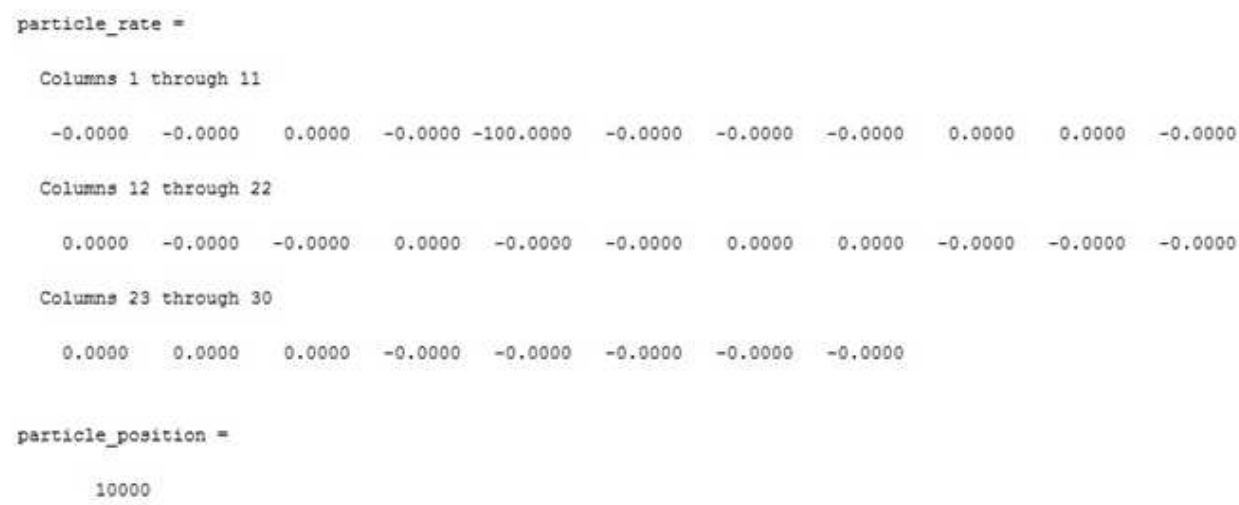

Fig. 2. Particle rate and particle position of each nodes 


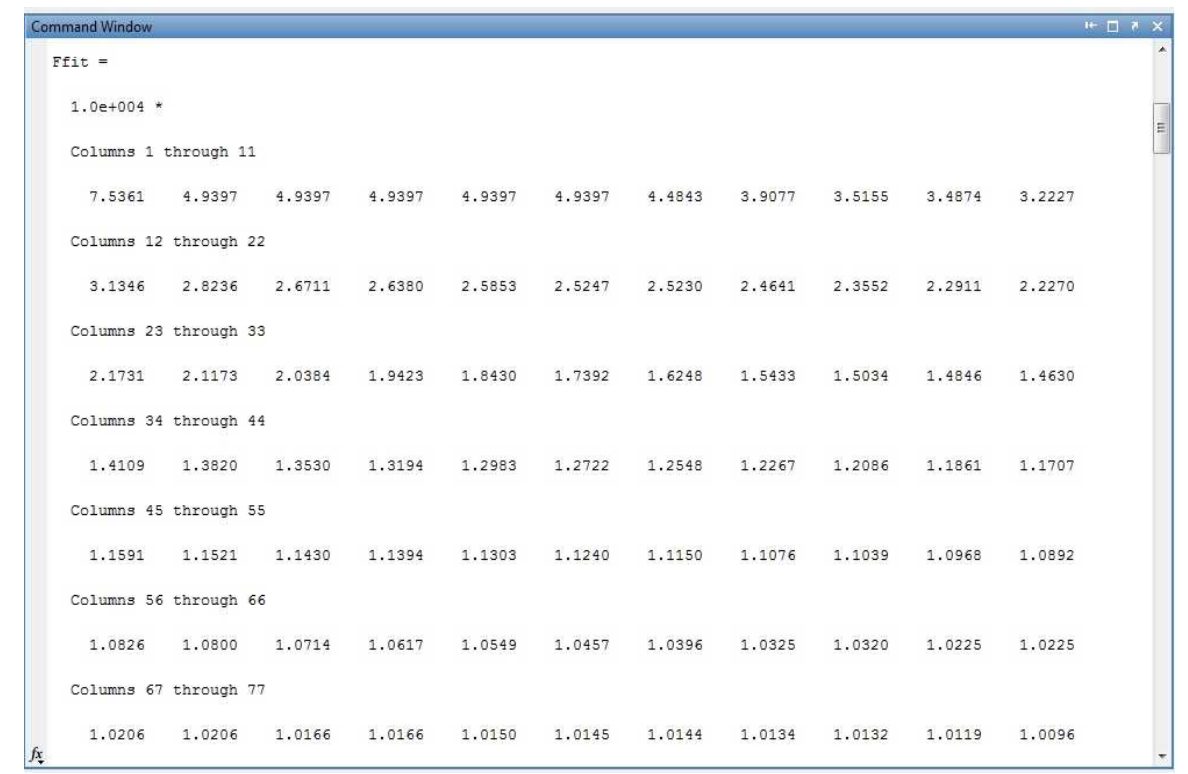

Fig. 3. Average analysis of FFit function

The source energy is set at $\mathrm{S}=5000, \mathrm{c} 1=\mathrm{c} 2=2$ and 17 are random numbers uniformly distributed in $[0,1]$. We conduct the trials with particle swarm optimization algorithm when the number of sensor in wireless sensor network is 20 and Signal-Noise-Ratio (SNR) is $40 \mathrm{db}$. The results are shown in Fig. 1 which clearly denotes the estimation error and connected matrix values of various algorithms (PSO-GA, PSO, GA). In this method, the estimated position of PSO-GA algorithm is much close to the actual source position, the estimation error between the actual position and the estimated position is about $1.3 \mathrm{~m}$ at each hop in WSN. The estimated position will approach to be the actual position with the increasing number of sensor nodes and signal-noise-ratio through the information of experiments and while the number of sensor nodes is small the estimated position of PSO algorithm is more accurately than other searching algorithms, in this case PSO-GA algorithm is used to obtain more accurate results with increased number of hop counts (sensor nodes).

We have performed lots of simulations to examine the performance of the proposed algorithm and other two algorithms. Different route algorithm and SignalNoise-Ratio (SNR) will affect the localization error.

The relationship between the estimation error of three localization algorithms and node number is shown in connection matrix as represented in Fig. 4 and the relationship between the estimation error of three localization algorithms and signal-noise-ratio is shown in Fig. 5.
From the Fig. 1, it is clearly denotes the estimation error of three methods; all decreases with increasing the number of sensor nodes in the wireless sensor network and the estimation error of PSO-GA is the least. The estimation error reduces rapidly while the nodes is more than 20 , but when the number of network nodes reach 40 the estimation error doesn't change in evidence, therefore, increasing the nodes isn't meaningful for enhancing the localization accuracy and which will increase the load of networks.

Figure 5 denotes clearly PSO-GA reaches a little estimation error with the reduced estimation of SNR. Compared to other algorithms PSO-GA has higher anti interference ability. However when SNR is large enough, it has little effect on improving proposed method performance. Therefore it is reasonable to set SNR to 30. This explains why the SNR is chosen respectively to be 30 and 50 in carrying out source localization. In this study, the computational complexity reflects the relationship of energy consumption indirectly because all of the three energy-based acoustic source localization methods are centralized algorithms with the same energy efficient communication utilization schemes. In the simulation; the size of the particle swarm is fixed at 20, multiresolution searching style is $4 * 4+25 * 25$ and the step of exhaustive search method is 2 and the number of iteration is $(100 / 2)^{*}(100 / 2)$. The iteration of three algorithms is shown in Table 1. 


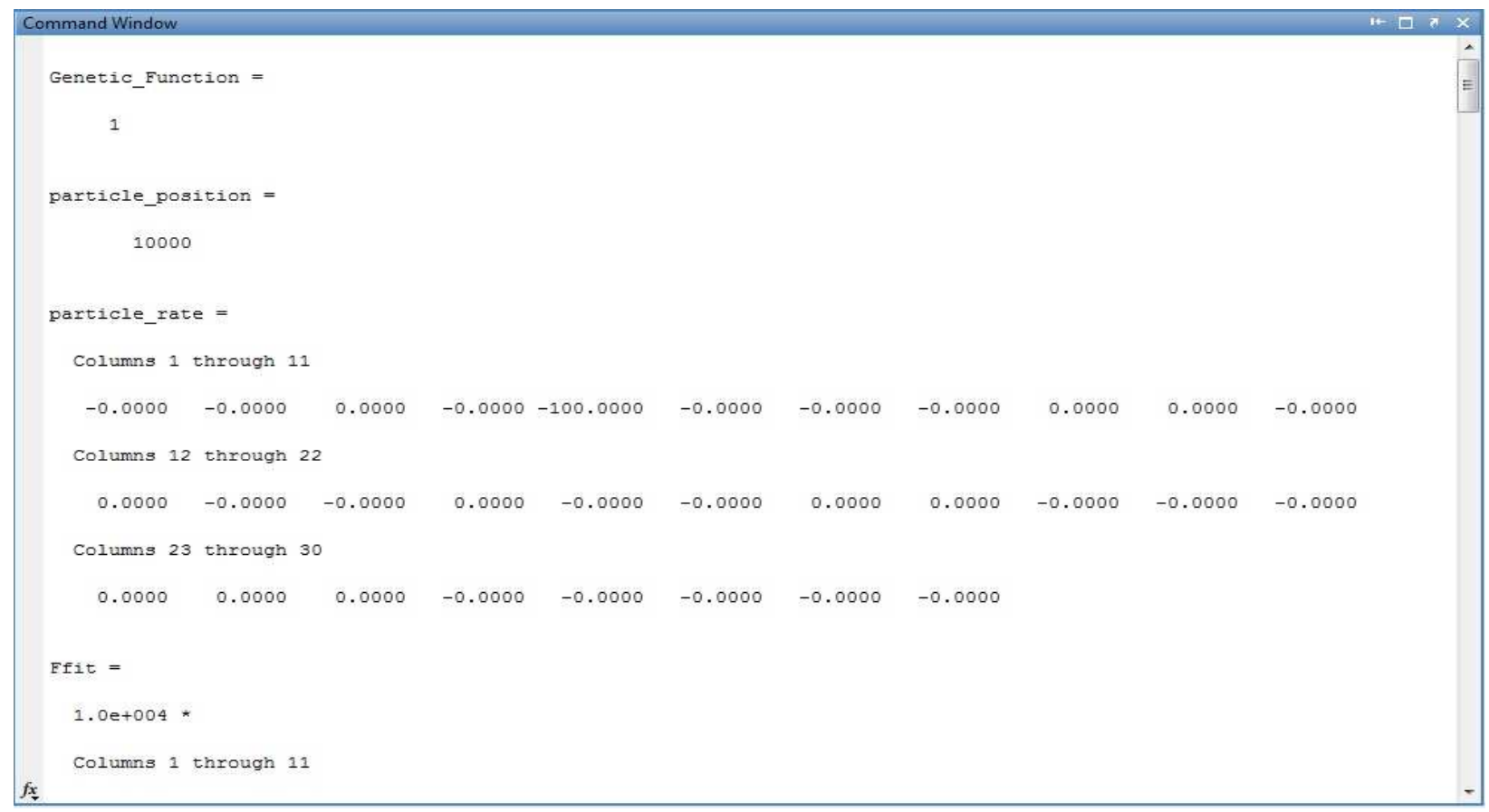

Fig. 4. Implementation evaluation PSO-GA algorithm

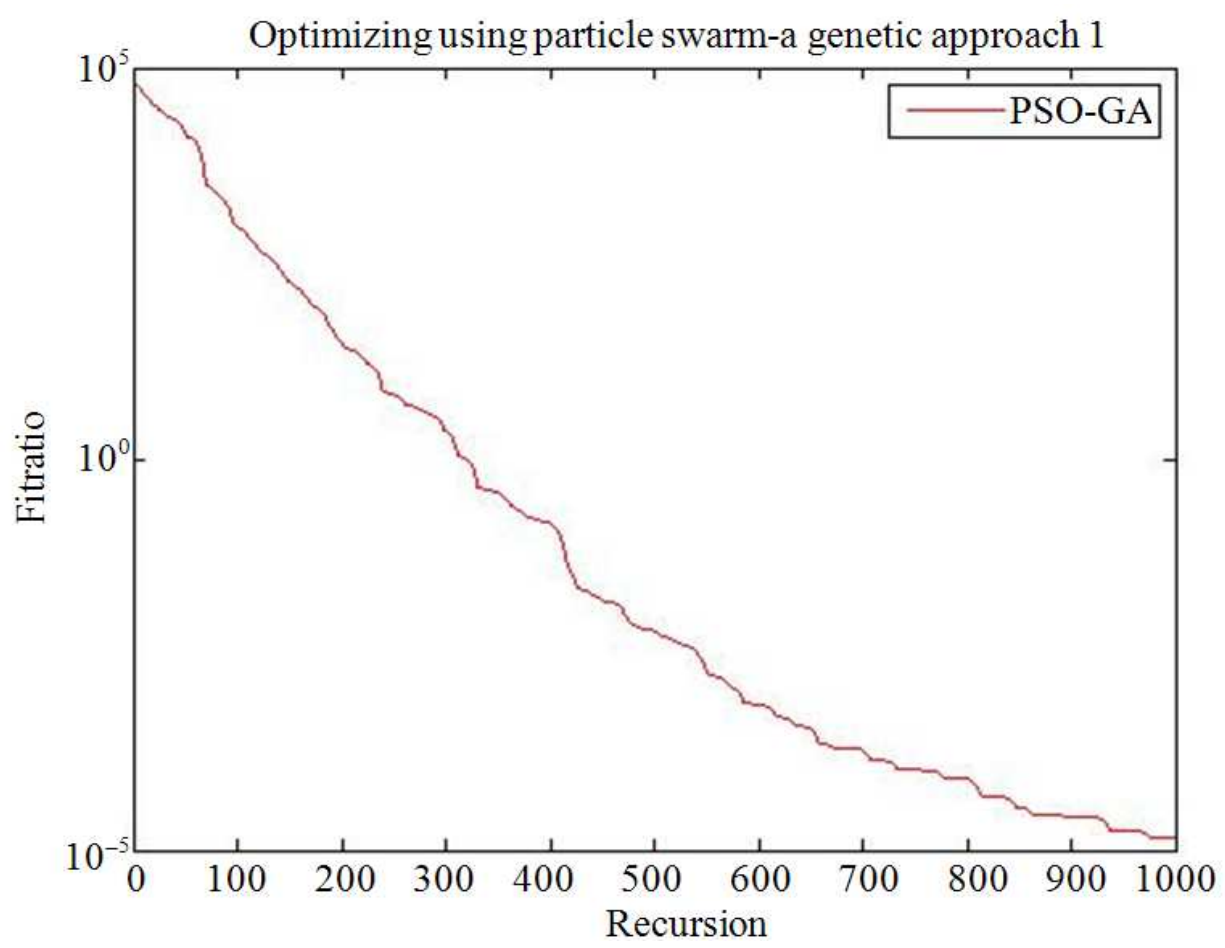

Fig. 5. Results of PSO-GA algorithm 
Table 1. Denotes the experimental result for Genetic, PSO, PSO-enetic (PSO-GA)

\begin{tabular}{|c|c|c|c|c|c|c|}
\hline \multirow[b]{2}{*}{ Dimension } & \multicolumn{2}{|l|}{ Genetic } & \multicolumn{2}{|l|}{ PSO } & \multicolumn{2}{|c|}{ Genetic-PSO } \\
\hline & Worst case & Best case & Worst case & Best case & Worst case & Best case \\
\hline 40 & -60 & $1.33 e-5$ & 0.7866 & $1.33 \mathrm{e}-60$ & 0.9453 & 0.3458 \\
\hline 60 & -60 & $1.33 e-5$ & 0.7866 & $1.33 \mathrm{e}-6$ & 0.9453 & 0.3458 \\
\hline 80 & -60 & $1.33 e-5$ & 0.7866 & $1.33 \mathrm{e}-6$ & 0.9453 & 0.3458 \\
\hline 100 & -60 & $1.33 e-5$ & 0.7866 & $1.33 \mathrm{e}-6$ & 0.9453 & 0.3458 \\
\hline 120 & $8.7 e-73$ & $1.33 e-5$ & -118 & -120 & $-05 \mathrm{e} 3.92$ & $1.97 \mathrm{e}-106$ \\
\hline 140 & $8.7 \mathrm{e}-73$ & $1.33 e-5$ & -118 & -120 & $-05 \mathrm{e} 3.92$ & $1.97 \mathrm{e}-106$ \\
\hline 160 & $8.7 \mathrm{e}-73$ & $1.33 e-5$ & -118 & -120 & $-05 \mathrm{e} 3.92$ & $1.97 \mathrm{e}-106$ \\
\hline 180 & $8.7 \mathrm{e}-73$ & $1.33 e-5$ & -118 & -120 & $-05 \mathrm{e} 3.92$ & $1.97 \mathrm{e}-106$ \\
\hline 200 & $8.7 e-73$ & $1.33 e-5$ & -118 & -120 & $-05 \mathrm{e} 3.92$ & $1.97 \mathrm{e}-106$ \\
\hline 220 & 2.0946 & $1.33 e-5$ & 3.8403 & $1.33 \mathrm{e}-60$ & $-05 \mathrm{e} 3.92$ & $1.97 \mathrm{e}-106$ \\
\hline 240 & 2.0946 & $1.33 e-5$ & 3.8403 & $1.33 e-6$ & $-05 \mathrm{e} 3.92$ & $1.97 \mathrm{e}-106$ \\
\hline 260 & 2.0946 & $1.33 e-5$ & 3.8403 & $1.33 \mathrm{e}-6$ & $3.54 \mathrm{e}-01$ & $1.98 \mathrm{e}-41$ \\
\hline 280 & -172 & $2.18 \mathrm{e}-10$ & 3.8403 & $1.33 \mathrm{e}-6$ & $3.54 \mathrm{e}-01$ & $1.98 \mathrm{e}-41$ \\
\hline 300 & -178 & $2.18 \mathrm{e}-10$ & 3.8403 & $1.33 e-60$ & $3.54 \mathrm{e}-01$ & 0.3458 \\
\hline 320 & -172 & $2.18 \mathrm{e}-10$ & 3.8403 & $1.33 \mathrm{e}-6$ & $3.54 \mathrm{e}-01$ & 0.3458 \\
\hline 340 & -60 & $1.97 \mathrm{e}-41$ & 3.8403 & $1.33 e-6$ & $3.54 \mathrm{e}-01$ & 0.3458 \\
\hline 360 & -60 & $1.33 e-5$ & $2.18 \mathrm{e}-14$ & $1.33 \mathrm{e}-6$ & $3.54 \mathrm{e}-01$ & 0.3458 \\
\hline 380 & -60 & $1.33 e-5$ & $2.18 \mathrm{e}-14$ & $8.7 e-73$ & $6.40 \mathrm{e}-30$ & 0.3458 \\
\hline 400 & -60 & $1.33 e-5$ & $2.18 \mathrm{e}-14$ & $8.7 \mathrm{e}-73$ & $6.40 \mathrm{e}-30$ & 0.3458 \\
\hline 420 & -60 & $1.33 e-5$ & $2.18 \mathrm{e}-14$ & $8.7 e-73$ & $6.40 \mathrm{e}-30$ & 0.3458 \\
\hline 440 & -50 & $2.18 \mathrm{e}-10$ & 6.8743 & $1.33 e-60$ & $6.40 \mathrm{e}-30$ & 0.3458 \\
\hline 460 & 2.0946 & $2.18 \mathrm{e}-10$ & 6.8743 & $1.33 \mathrm{e}-6$ & $6.40 \mathrm{e}-30$ & $4.85 \mathrm{e}-07$ \\
\hline 480 & 2.0946 & $2.18 \mathrm{e}-10$ & 6.8743 & $1.33 \mathrm{e}-6$ & $8.7 e-73$ & $4.85 \mathrm{e}-07$ \\
\hline 500 & 2.0946 & $1.97 \mathrm{e}-41$ & $2.18 \mathrm{e}-10$ & $1.33 \mathrm{e}-6$ & $8.7 \mathrm{e}-73$ & $4.85 \mathrm{e}-07$ \\
\hline
\end{tabular}

Table 2. Recursion ratio for PSO-GA,MR,Exhaustic research

\begin{tabular}{llll}
\hline Complexity & PSO-GA & MR & Exhaustive research \\
\hline Recursion ratio & 400 & 641 & 2500 \\
\hline
\end{tabular}

From the Table 2 derived from Table 1, it is noted that the computational complexity of PSO-GA algorithm is lowest and it has the highest localization precision with the same parameters in same trail. For MR and exhaustive search algorithms, enhancing localization precision must be at the cost of increasing computational complexity because of their computational method based on iteration. Compared to other conventional optimization methods PSO-GA has lots of advantages such as it hasn't special requirements at the form of objective function which makes it have extensive application. At the same time based on the characteristics of evolution for the probability which ensures the rapidity of the method, so for the optimization of complex computational problem, PSOGA has a strong advantage.

\subsection{Future Enhancement}

In this study, we achieved an accuracy level of $81 \%$ in terms of switching our PSO context towards GA, since GA is one of the exceptional domain which works on Heuristic principles, since the exposed idea reveals not much more than $81 \%$ in terms of performance, since to increase the accuracy level of optimizing the route in WSN, adhoc or in VANET, our methodology is to be extended to achieve higher accuracy. In future we would like to extend our work in MANET with better accuracy than the previous.

\section{CONCLUSION}

In this study, a particle swarm optimization for optimizing the route based on PSO-GA algorithm for wireless sensor network has been proposed. The localization problems are optimized by using the presented method and have been evaluated, validated with extensive simulation study which consistently promises superior performance and is easy to implement; as compared with MR and exhaustive searching localization methods. From the study, the results have demonstrated that the proposed approach has higher precision and lower computational complexity in source 
localization for the wireless sensor network. Results show that the proposed algorithm is better than general PSO and GA. The correlation value of PSO-GA lies on 0.99978 (approximately).

\section{REFERENCES}

AlfassioGrimaldi, E., F. Grimaccia, M. Mussetta and P. Pirinoli et al., 2004. A new hybrid genetical-swarm algorithm for electromagnetic optimization. Proceedings of the 3rd International Conference on Computational Electromagnetics and Its Applications, Nov. 1-4, IEEE Xplore Press, pp: 157160. DOI: 10.1109/ICCEA.2004.1459314

Arabas, J., Z. Michalewicz and J. Mulawka, 1994. GA Vs PS-A genetic algorithm with varying population size. Proceedings of IEEE International Conference on Evolutionary Computation, (EC' 94), Orlando, pp: 73-78.

Boeringer, D.W. and D.H. Werner, 2004. Particle swarm optimization versus genetic algorithms for phased array synthesis. IEEE Trans. Antennas Propagat., 52: 771-779. DOI: 10.1109/TAP.2004.825102

Ciuprina, G., D. Loan and I. Munteanu, 2002. Use of intelligent-particle swarm optimization in electromagnetic. IEEE Trans. Magn., 38: 10371040. DOI: $10.1109 / 20.996266$

Clerc, M. and J. Kennedy, 2002. The particle swarmexplosion, stability and convergence in multidimensional complex space. IEEE Trans. Evolut. Comput., 6: 58-73. DOI: 10.1109/4235.985692

Goldberg, D.E., 1989. Genetic Algorithms in Search, Optimization and Machine Learning. 1st Edn., Addison-Wesley, New York, ISBN-10: 0201157675, pp: 432.

Grimaccia, F., M. Mussetta and R.E. Zich, 2006. Comparison of different hybridization strategies in evolutionary optimization for EM. Proceedings of Antennas and Propagation Society International Symposium, Jul. 9-14, IEEE Xplore Press, Albuquerque, NM, pp: 585-588. DOI: 10.1109/APS.2006.1710591

Juang, C.F., 2004. A hybrid of genetic algorithm and particle swarm optimization for recurrent network design. IEEE Trans. Syst. Man Cybernet., 34: 997-1006. DOI: 10.1109/TSMCB.2003.818557

Kennedy, J., 1997. The particle swarm: Social adaptation of knowledge. Proceedings of the IEEE International Conference on Evolutionary Computation, Apr. 1316, IEEE Xplore Press, Indianapolis, IN., pp: 303308. DOI: 10.1109/ICEC.1997.592326
Lee, S. and D. Knignt, 2005. Realization of next generation network. IEEE Commun. Mag., 43: 3441. DOI: 10.1109/MCOM.2005.1522122

Murthy, C.S.R. and B.S. Manoj, 2004. Ad Hoc Wireless Networks: Architectures and Protocols. 1st Edn., Pearson Education, Upper Saddle River, ISBN-10: 0132465698, pp: 879.

Mussetta, M., G. Dassano, P. Pirinoli, R.E. Zich and M.Orefice, 2004. Design, optimization and experimental measurements of a $18 \mathrm{GHz}$ microstrip reflectarray. Proceedings of the 13th International Symposium on Antennas, JINA, Nov. 8-10, Nice, France, pp: 240-241.

Mussetta, M., P. Pirinoli, R.E. Zich and M. Orefice, 2005. Design of printed microstrip reflect arrays reducing the ground plane reflection. Proceedings of the IEEE 28th URSI General Assembly, (GA' 05), pp: 23-29.

Osama, H.H., N.S. Tarek and J.L. Myung, 2005. Probability routing algorithm for mobile ad hoc networks' resources management. IEEE J. Selected Areas Commun., 23: 2248-2259. DOI: 10.1109/JSAC.2005.857205

Rahmat-Samii, Y. and E. Michielssen, 1999. Electromagnetic Optimization by Genetic Algorithms. 1st Edn., Wiley, New York, ISBN-10: 0471295450, pp: 480.

Robinson, J., S. Sinton and Y. Rahmat-Samii, 2002. Particle swarm, genetic algorithm and their hybrids: Optimization of a profiled corrugated horn antenna. Proceedings of the IEEE International Symposium on Antennas and Propagation, Jun. 16-21, IEEE Xplore Press, pp: 314-317. DOI: 10.1109/APS.2002.1016311

Rong, H.U., H.U. Zhigang and M.A. Hao, 2005. A reliable routing algorithm based on fuzzy applicability of F sets in MANET. Proceedings of the 11th Pacific Rim International Symposium on Dependable Computing, Dec. 12-14, IEEE Xplore Press. DOI: 10.1109/PRDC.2005.13

Tanese, R., 1989. Distributed genetic algorithm. Proceedings of the International Conference on Genetic Algorithms, (CGA' 89), pp: 434-439.

Visu, P. and E. Kannan, 2013. Combined swarm intelligence routing protocol for MANET. Inform. Technol. J., 12: 791-797. DOI: 10.3923/itj.2013.791.797

Visu, P., S. Koteeswaran and J. Janet, 2012. Artificial bee colony based energy aware and energy efficient routing protocol. J. Comput. Sci., 8: 227-231. DOI: 10.3844/jcssp.2012.227.231 